\title{
Room-temperature ferromagnetic and photoluminescence properties of indium-tin-oxide nanoparticles synthesized by solid-state reaction
}

\author{
S HARINATH BABU ${ }^{1}$, N MADHUSUDHANA RAO ${ }^{1}$, S KALEEMULLA ${ }^{1, *}$, G AMARENDRA ${ }^{2,3}$ \\ and C KRISHNAMOORTHI ${ }^{4}$ \\ ${ }^{1}$ Thin Films Laboratory, Center for Crystal Growth, VIT University, Vellore 632 014, India \\ ${ }^{2}$ Materials Science Group, Indira Gandhi Centre for Atomic Research, Kalpakkam 603102, India \\ ${ }^{3}$ UGC-DAE-CSR, Kalpakkam Node, Kokilamedu 603104, India \\ ${ }^{4}$ Center for Nano Research, VIT University, Vellore 603104, India
}

MS received 10 March 2016; accepted 9 June 2016

\begin{abstract}
In the present study, indium-tin-oxide (ITO) nanoparticles were synthesized using solid-state reaction and studied for their structural, vibrational and magnetic properties. The ITO nanoparticles were prepared under reduced pressure, which can increase the oxygen vacancies in the samples. The $\mathrm{X}$-ray diffraction studies confirmed singe-phase cubic bixbyite structure of ITO with average crystallite size of $47 \mathrm{~nm}$. The lattice vibrational studies (FTIR and Raman spectroscopy) at room temperature indicated that $\mathrm{Sn}$ ions were occupied in $\operatorname{In}_{2} \mathrm{O}_{3}$ lattice and gives corresponding active vibrational modes in the respective spectra. The magnetic studies at room temperature reveal the ferromagnetic nature of ITO and the strength of magnetization is superior to those of $\mathrm{In}_{2} \mathrm{O}_{3}$ and $\mathrm{SnO}_{2}$. However, the magnetic studies at $100 \mathrm{~K}$ revealed reduced ferromagnetism, which could be attributed to reduced itinerary electrons at low temperature. Blue and blue-green emissions were found from the ITO nanoparticles, which could be due to vacancies or surface defects present in the system.
\end{abstract}

Keywords. Indium-tin oxide; solid-state reaction; ferromagnetism; photoluminescence.

\section{Introduction}

Development of good dilute magnetic semiconductors (DMSs) is necessary to prepare spintronic devices in which both spin and charge of the electrons are exploited [1]. The DMS materials can be developed by doping compatible transition metal (TM) ions into a conventional semiconductor [2]. In the past one and half decades many researchers developed DMS materials with various host semiconductors such as titanium dioxide, copper oxide, indium oxide, tin oxide, etc. [3-9]. The oxide-based DMS exhibited ferromagnetism at room temperature, whereas the alloy-based DMS exhibited ferromagnetism at lower temperatures. In addition, oxidebased DMSs are easy to synthesize compared with their counterpart alloy-based DMSs. Further, the former materials are cost-effective than the latter materials. However, the oxygen-induced ferromagnetism is feeble than that of transition-element-doped oxide DMS. Many semiconductors such as $\mathrm{ZnO}, \mathrm{SnO}_{2}, \mathrm{In}_{2} \mathrm{O}_{3}, \mathrm{TiO}_{2}$, etc. were studied to develop good oxide-based DMS materials and it was realized that the induced ferromagnetism in these materials at room temperature by TM doping is moderate and requires further improvement for spintronic applications. However, it is known that the ferromagnetic property of the semiconducting material depends upon deposition conditions such as method of preparation, oxygen deficiencies, vacuum or Ar ambience,

*Author for correspondence (skaleemulla@gmail.com) thickness, etc. If the films are prepared in oxygen-deficient environment, more magnetic moment will be observed. However, when the same films are annealed in air at higher temperatures, the magnetic moment will be decreased, indicating that the observed ferromagnetism is due to oxygen vacancies. Moreover, the oxide semiconductor exhibiting diamagnetism in bulk form became ferromagnetic in thin film form. Hence it is necessary to observe the ferromagnetism in bulk form and the change in its magnetic moment in thin film form. A few reports suggested that defects will also play an important role in magnetic properties of the host materials. Motivated by these contradictory results, in the present study we have synthesized indium-tin-oxide (ITO) nanoparticles and studied systematically to find the origin for the observed ferromagnetism.

We decided on feasibility of development of ITO-based DMS materials since ITO is a wide-band-gap material and its other properties are well understood in the literature [10]. Indium oxide $\left(\mathrm{In}_{2} \mathrm{O}_{3}\right)$ has found many applications such as in solar cells, flat panel displays and touch screens due to its high optical transparency and high electrical conductivity. In order to integrate the optical, electrical and magnetic properties in the spintronic devices, the materials suitable for such devices should have high tunability of charge carriers, high carrier mobility, high optical transparency, high abundance of elements and low impact on the environment. $\mathrm{In}_{2} \mathrm{O}_{3}$ is one of the most promising candidates for such tasks. When $\mathrm{In}_{2} \mathrm{O}_{3}$ is doped with a small quantity of tin ( $\mathrm{Sn})$, a so-called ITO is 
formed. The high conductivity of ITO is attributed to the high carrier concentration, which is caused by incorporation of $\mathrm{Sn}$ ions into $\operatorname{In}_{2} \mathrm{O}_{3}$ host lattice and creation of off-stoichiometry. It has a cubic bixbyite structure with the lattice parameter $a=10.118 \AA$. ITO is an n-type transparent conducting oxide (TCO), which is widely used in the manufacture of transparent electrodes, photoelectronic devices, photosensors, liquid crystal displays, electrochromic windows, solar panels and transparent coatings for solar-energy heat mirrors [11-13]. Here we report on magnetic properties of ITO nanoparticles at room temperature and at $100 \mathrm{~K}$.

\section{Experimental}

$\mathrm{In}_{1.9} \mathrm{Sn}_{0.1} \mathrm{O}_{3}$ powder samples were prepared from commercially available high-purity $\mathrm{In}_{2} \mathrm{O}_{3}$ and $\mathrm{SnO}_{2}$ both from Sigma-Aldrich, $99.999 \%$ precursors. The precursors were mixed in metal ion stoichiometric ratios and ground for $12 \mathrm{~h}$ to make very fine powder mixture. The mixture was placed into suitable quartz test tubes $(10 \mathrm{~mm})$ whose interior was enclosed in another quartz tube with evacuation and heating provision. These enclosed samples were evacuated to optimal pressure $\left(2 \times 10^{-3}\right)$ mbar by a rotary vane pump, heated at the rate of $5^{\circ} \mathrm{C} \mathrm{min}{ }^{-1}$ and soaked at $800^{\circ} \mathrm{C}$ for $8 \mathrm{~h}$.

$\mathrm{X}$-ray diffraction (XRD; X-ray diffractometer, D8 Advance, BRUKER) patterns were used to study the structural aspects. The microstructure and chemical composition analysis of the samples were carried out by field emission scanning electron microscopy (FE-SEM) with energy dispersive analysis of X-ray spectroscopy (EDAX; Oxford Instruments, INCA PentaFET-x3). The valence states of the elements were studied by X-ray photoelectron spectroscopy, XPS (K-Alpha, Thermoelectron). The photoluminescence spectrum of the sample was recorded using a spectrophotometer (Scinco FluoroMasterPlus fluorimeter) with excitation wavelength of
$300 \mathrm{~nm}$. The magnetic properties of the samples were studied through magnetic-field-dependent magnetization loops (hysteresis loops) up to $0.6 \mathrm{~T}$ field at room temperature $(300 \mathrm{~K})$ and at low temperature $(100 \mathrm{~K})$ using a calibrated vibrating sample magnetometer (VSM, Lake Shore 7303).

\section{Results and discussion}

The XRD profiles of $\mathrm{SnO}_{2}$ and $\mathrm{In}_{2} \mathrm{O}_{3}$ powders were also studied but are not shown here. Figure 1 shows the XRD profile of the precursors as well as synthesized ITO powders. The diffraction peaks in $\mathrm{SnO}_{2}$ profile could be assigned to different Bragg's planes as follows: $26.62^{\circ}\left(\begin{array}{lll}1 & 1 & 0\end{array}\right), 33.89^{\circ}$ (1 01 1), 37.98 $(200), 39.09^{\circ}(111), 42.65^{\circ}(210), 51.80^{\circ}$ (2 11 1), $54.79^{\circ}(220), 57.85^{\circ}(002), 61.92^{\circ}(310), 64.75^{\circ}$ (1 12 2), $65.98^{\circ}$ (3 01 1), $71.39^{\circ}$ (2 02 ) and $78.73^{\circ}$ (3 21 ) according to tetragonal structure of $\mathrm{SnO}_{2}$ [JCPDS No. 411445]. The peaks at different angles in ITO profile could be assigned to different Bragg's planes as follows: (2 11 1), (2 22 2), (4 00 ),

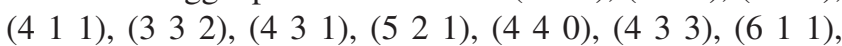
(5 4 1), (6 22 2), (6 3 1), (4 44 4), (5 4 3), (6 40 ) and (7 21 1) according to the cubic structure of $\mathrm{In}_{2} \mathrm{O}_{3}$ (JCPDS No. \#060416) and no other crystal planes were found in the synthesized ITO powders. However, the intensity of the diffraction peaks decreases and diffraction peaks shifted towards higher angles when $\mathrm{Sn}$ was doped into $\operatorname{In}_{2} \mathrm{O}_{3}$ lattice. This is clearly shown in the inset of figure 1 . The estimated lattice constant of $\operatorname{In}_{2} \mathrm{O}_{3}$ is $10.112 \AA$ and it is reduced to $10.08 \AA$ in ITO fine powders. A lattice constant of $10.112 \AA$ was also observed in bulk ITO by Xia et al [14]. This may be attributed to the fineness of our samples.

The crystallite size $(G)$ was calculated using the DebyeScherrer formula [15]

$$
G=k \lambda / \beta \cos \theta,
$$

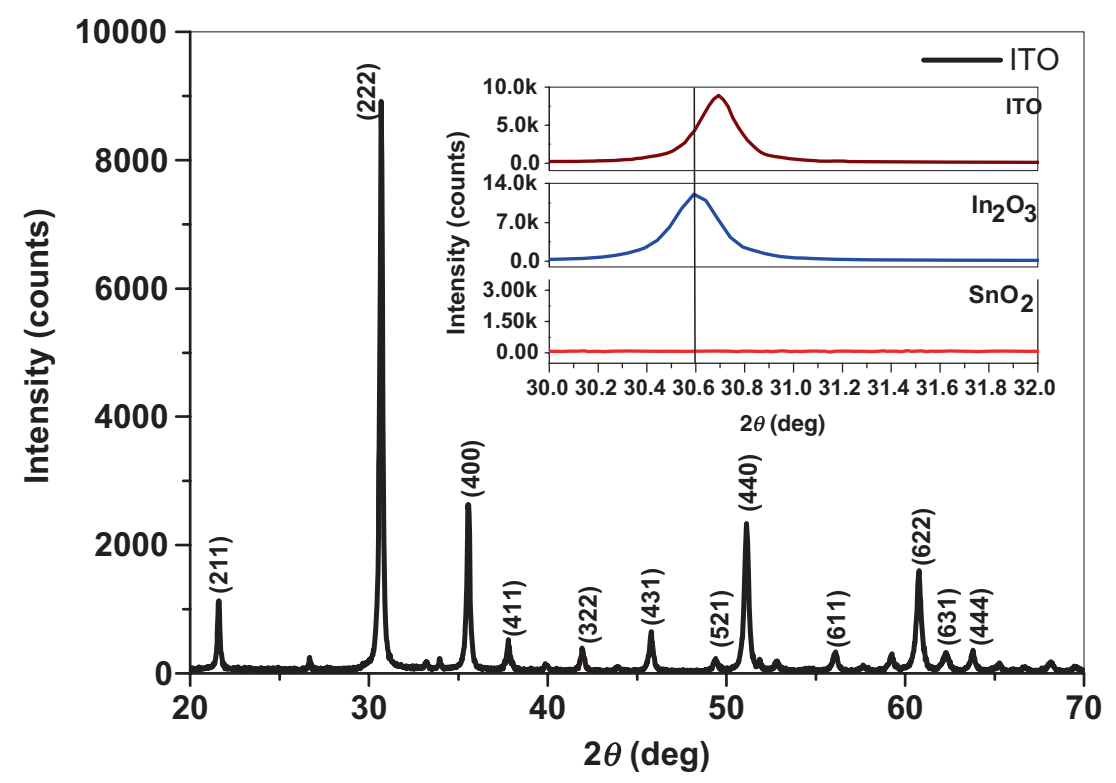

Figure 1. X-ray diffraction profile of ITO nanoparticles. 
where $k$ is a particle-geometry-dependent constant (for spherical shape $k \sim 1), \lambda$ is the wavelength used $(\lambda=$ $1.5406 \AA$ ), $\beta$ is the full-width at half-maximum (FWHM) and $\theta$ is the diffracted angle. The estimated average crystallite size is found to be $47 \mathrm{~nm}$. In order to confirm the nanocrystallinity of the ITO powders, room-temperature FE-SEM micrographs of ITO powders are shown.

Figure 2 shows a FE-SEM micrograph of the ITO fine powders. The micrograph shows well-defined particles with varied geometries formed by flocculation and aggregation. A few particles show elongated geometry with aspect ratio $>1.5$. The average particle size estimated from the micrograph is $65 \mathrm{~nm}$, which is higher than the crystallite size estimated from XRD profile. Hence we infer that the particles are multi-grained.

Figure 3 shows representative EDAX spectrum of ITO fine powder collected by EDAX attachment in the FE-SEM instrument. The EDAX spectrum shows all the constituent elements in ITO fine powder and is close to the targeted metal composition. However, oxygen content is less than the target value.

To elucidate the valence state of the In, Sn and O elements in ITO fine powders, XPS analysis was carried out and the results are shown in figure 4 . The In $3 \mathrm{~d}$ peaks were observed at binding energies of 444.14 and $451.62 \mathrm{eV}$, which represent the valence state of indium in $\mathrm{In}^{3+}$ states. No peak

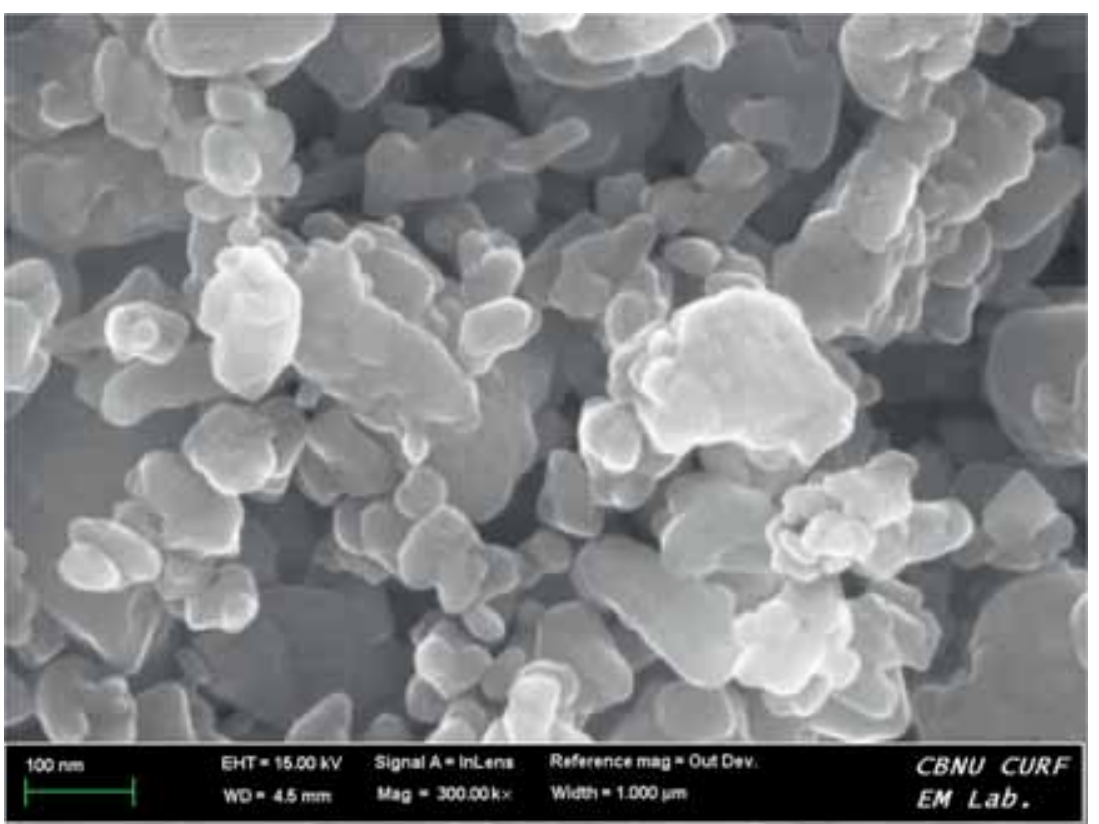

Figure 2. FE-SEM image of ITO nanoparticles.

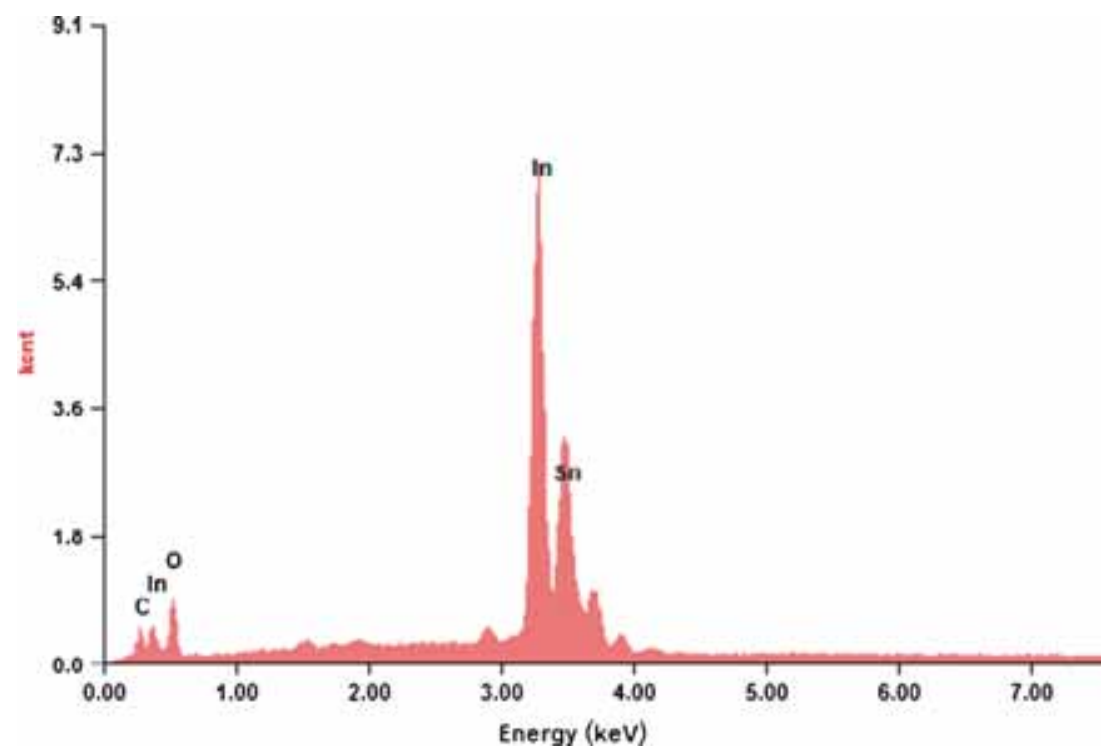

Figure 3. EDAX spectrum of ITO nanoparticles. 


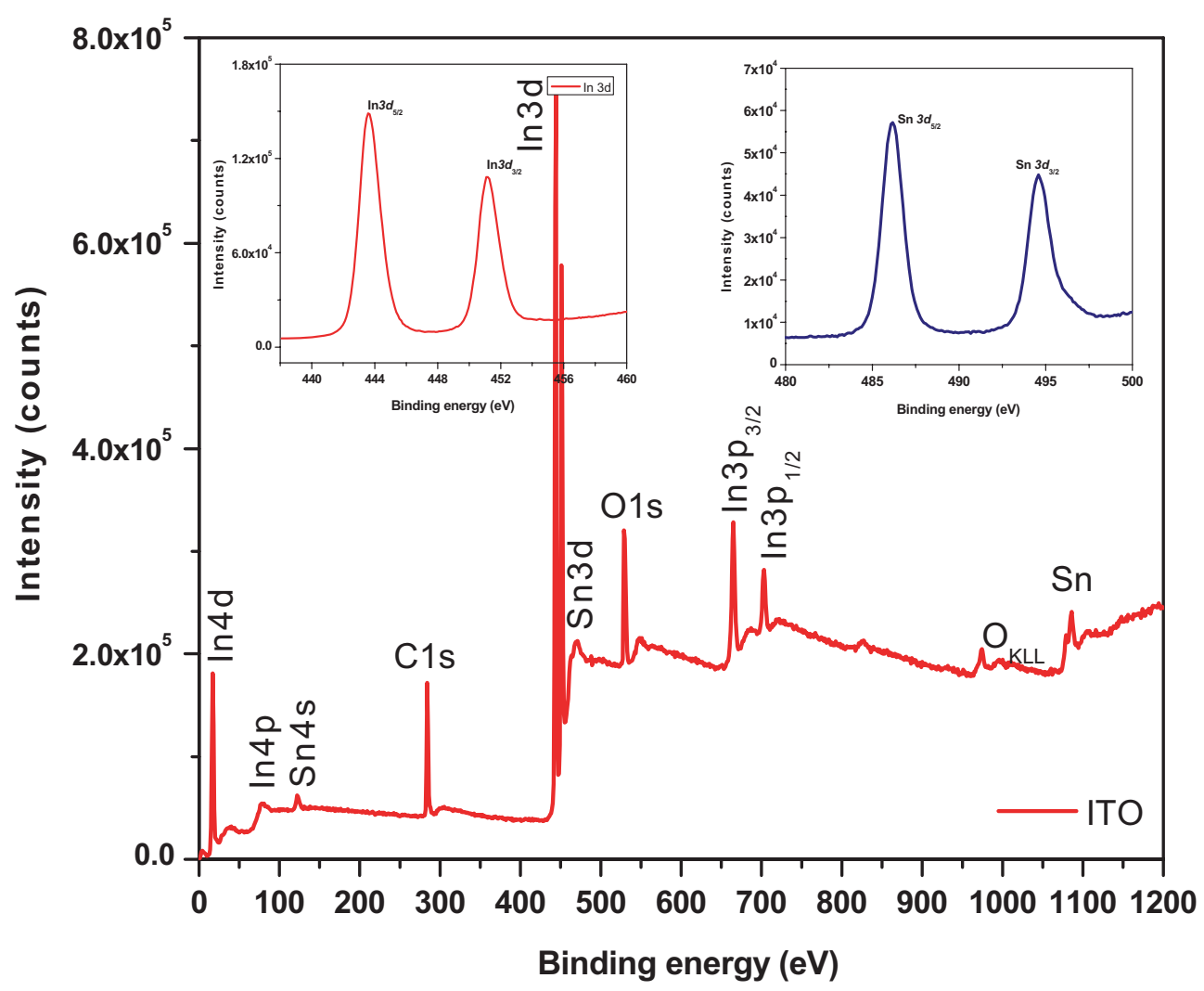

Figure 4. Survey scans of XPS spectra of ITO nanoparticles. The inset of the figure shows the XPS fine spectra of indium and tin elements.

related to metal indium $\operatorname{In}^{0}$ with binding energy $443.6 \mathrm{eV}$ was observed. The binding energies of Sn3d were found at 486.15 and $494.58 \mathrm{eV}$. The position of the Sn3d peaks fitted well with the reported binding energy of $\mathrm{SnO}_{2}(486.4 \mathrm{eV})[16,17]$. The $\mathrm{O} 1 \mathrm{~s}$ binding energy peak was observed at $531.67 \mathrm{eV}$. The O1s peak coincides well with the expected binding energy of $\mathrm{In}_{2} \mathrm{O}_{3}$ and $\mathrm{SnO}_{2}(530.5$ and $530.6 \mathrm{eV})$. Similar results were also observed in ITO thin films by Majumdar et al [18]. From the survey scan it was found that no other metallic impurities, especially ferromagnetic, were found in ITO within the detection limit of XPS instrument. It suggests that the observed room-temperature ferromagnetism in ITO powders is due to its intrinsic nature rather than any metal clusters.

FT-IR spectroscopic analysis was carried out in order to observe the bonding in ITO fine powders. Figure 5 shows the FT-IR spectrum of the ITO powders between 4000 and $400 \mathrm{~cm}^{-1}$. The bands observed in the wavenumber range of $430-620 \mathrm{~cm}^{-1}$ are related to the vibration of antisymmetric O-Sn-O bridge functional groups of $\mathrm{SnO}_{2}$. The In$\mathrm{O}$ stretching in cubic $\operatorname{In}_{2} \mathrm{O}_{3}$ nanopowder had intense bands at 440 and $557 \mathrm{~cm}^{-1}$ as reported by Ayeshamariam et al [19]. The $\mathrm{In}_{2} \mathrm{O}_{3}$ powder exhibited intense peaks at 538 and $565 \mathrm{~cm}^{-1}$. The absorption bands arising below the wavenumber $1000 \mathrm{~cm}^{-1}$ in metal oxides are attributed to the interatomic vibrations and additional peaks obtained above the wavenumber $1000 \mathrm{~cm}^{-1}$ could be due to the overtones. The FT-IR spectra exhibit the characteristic signatures of $\mathrm{SnO}_{2}$ of the $\mathrm{Sn}-\mathrm{O}$ bond with absorption peaks between 430 and
$620 \mathrm{~cm}^{-1}$, whereas the cubic $\mathrm{In}_{2} \mathrm{O}_{3}$ powder had intense bands of the In-O stretching at the wavenumber of 440 and $557 \mathrm{~cm}^{-1}$.

Raman spectroscopy is a commonly used technique to study doping effects in doped semiconductor nanocrystals as shifts in lattice Raman vibrational energies occur with increasing dopant concentration. Figure 6 shows the Raman spectra of $\mathrm{SnO}_{2}, \mathrm{In}_{2} \mathrm{O}_{3}$ and ITO powders. As a proof of cation substitution, we measured the unpolarized Raman spectra of ITO nanoparticles to investigate the effects of the substitution of $\mathrm{Sn}$ ions in the $\mathrm{In}_{2} \mathrm{O}_{3}$ host lattice. In the Raman spectra, the pure $\mathrm{SnO}_{2}$ spectrum showed the $\mathrm{SnO}_{2}$ vibrations at 568 and $630 \mathrm{~cm}^{-1}$, which can be attributed to the A1g mode. Similar Raman peaks were observed in nanocrystalline $\mathrm{SnO}_{2}$ powder with average grain size of about $4 \mathrm{~nm}$ prepared by Shek et al [20] via sol-gel method and Kaur et al [21] in ITO nanoparticles. Raman-active peaks at 125, 475,631 and $773 \mathrm{~cm}^{-1}$, corresponding to the $\mathrm{B} 1 \mathrm{~g}, \mathrm{Eg}, \mathrm{A} 1 \mathrm{~g}$ and $\mathrm{B} 2 \mathrm{~g}$ vibration modes, respectively, were observed, and are in good agreement with those for the rutile bulk $\mathrm{SnO}_{2}$ observed by Mcguire et al [22]. A1g and B2g modes are due to the expanding and contracting vibration of $\mathrm{Sn}-\mathrm{O}$ bonds, respectively [23]. From the figure it can be seen that the characteristic Raman peaks of $\operatorname{In}_{2} \mathrm{O}_{3}$ lying at 110, 132, 154, $164,212,249,307,365,480,495$ and $631 \mathrm{~cm}^{-1}$ are in good agreement with the results reported by Dussan et al [24]. The observed Raman bands around 306, 365, 494 and $628 \mathrm{~cm}^{-1}$ are assigned to phonons associated with the bcc-structured 


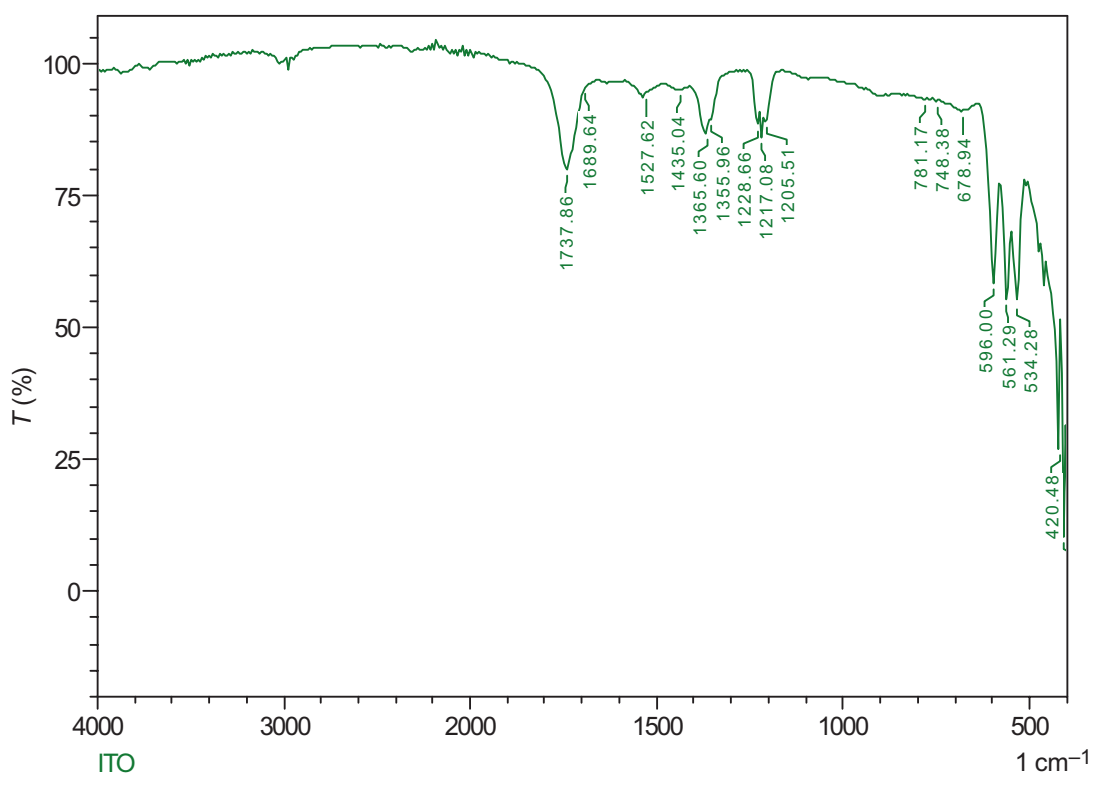

Figure 5. FT-IR spectrum of ITO nanoparticles.

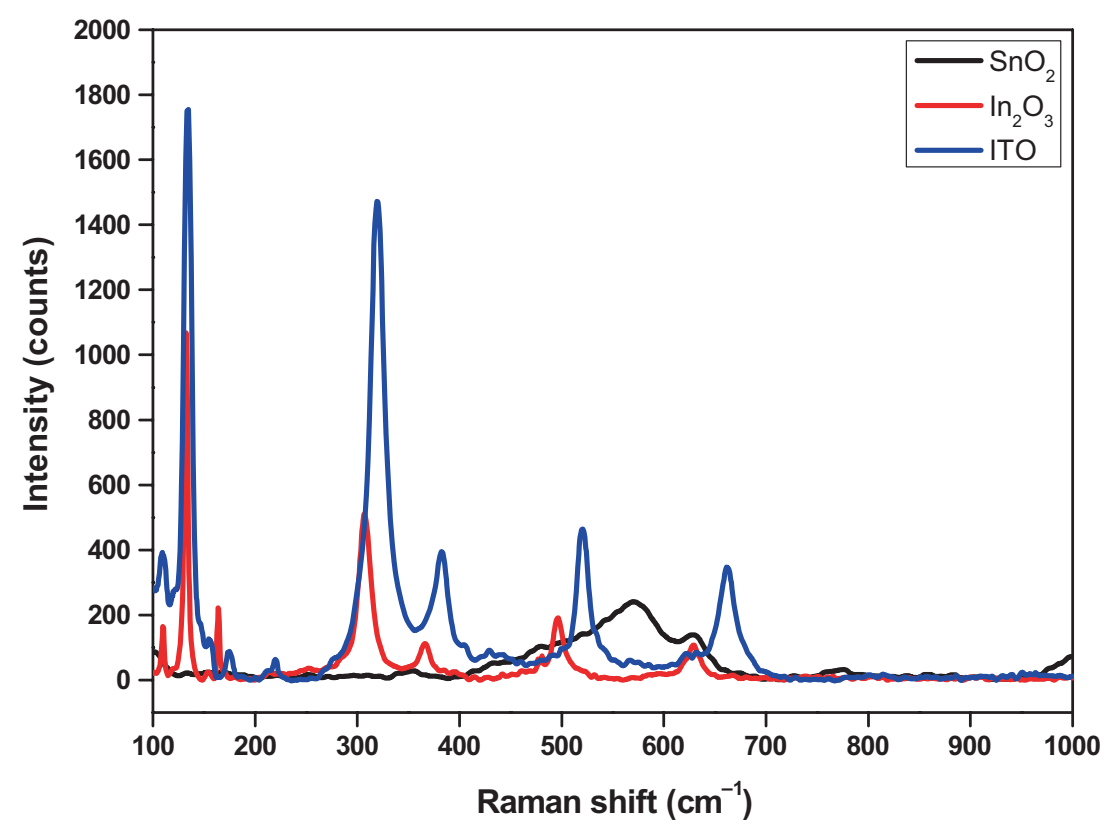

Figure 6. Raman spectra of $\mathrm{In}_{2} \mathrm{O}_{3}$ and ITO nanoparticles.

indium oxide. Similar Raman peaks were also observed in ITO fine powders. But a blue-shift in Raman peaks was observed. It may be due to substitution of Sn into $\mathrm{In}_{2} \mathrm{O}_{3}$ lattice. The density of oxygen vacancies increases with increase of dopant concentration. Similar results were also observed in Co-doped $\mathrm{TiO}_{2}$ nanoparticles reported by Santara et al [25].

Figure 7 shows the magnetic hysteresis loop of ITO powders at room temperature after subtracting the diamagnetic or paramagnetic background. The ITO powders exhibited the saturation magnetic moment, coercivity and retentivity of $0.05 \mathrm{emu} \mathrm{g}^{-1}, 683 \mathrm{G}$ and $0.02 \mathrm{emu} \mathrm{g}^{-1}$, respectively, at room temperature. Since there are no other ferromagnetic impurities in the sample, these observations suggest that the origin of magnetism in these samples must be due to the oxygen vacancies that are created during synthesis under reduced pressure. The experimental results further suggest that the ferromagnetism is an intrinsic property of ITO and does not arise from any metallic impurities in ITO. Our sample was prepared in vacuum, and hence we can expect oxygen vacancies. Figure 7 shows the magnetic hysteresis curves of ITO powders at 300 and $100 \mathrm{~K}$. It seems that the ferromagnetism is favourable in ITO at room temperature rather than $100 \mathrm{~K}$. The origin of room-temperature ferromagnetism 


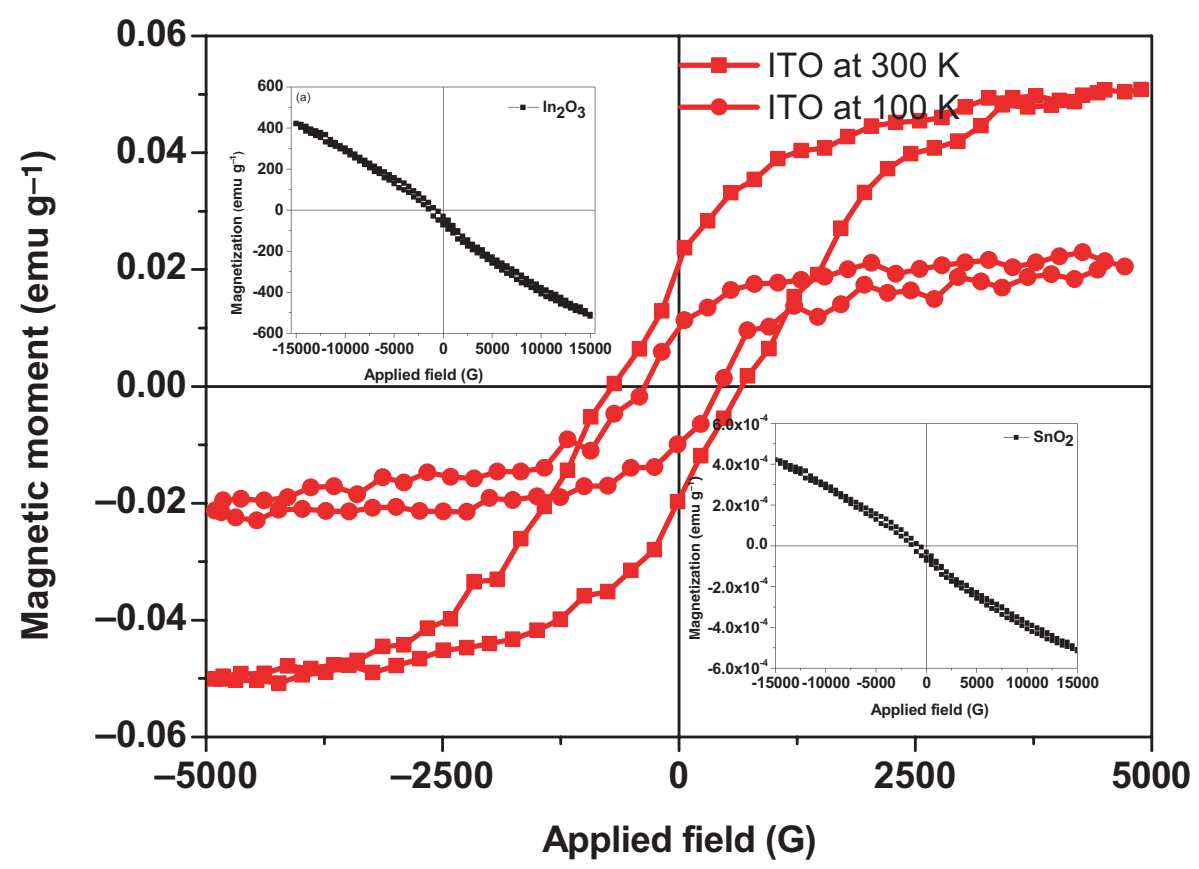

Figure 7. Magnetic hysteresis loops $(M-H)$ of ITO nanoparticles at 300 and $100 \mathrm{~K}$. The top and bottom insets of the figure shows the diamagnetic nature of, respectively, bulk $\operatorname{In}_{2} \mathrm{O}_{3}$ and $\mathrm{SnO}_{2}$.

in oxide semiconductor was explained by carrier-mediated ferromagnetism [26,27] and bound magnetic polaron model $[28,29]$. The doped $\operatorname{In}_{2} \mathrm{O}_{3}$ and $\mathrm{SnO}_{2}$ nanoparticles exhibited diamagnetic nature, whereas 10-at\%-Sn-doped $\operatorname{In}_{2} \mathrm{O}_{3}$ exhibited a clear ferromagnetic hysteresis loop. Hence it may be considered that carrier-mediated ferromagnetism may be one possible origin for the observed room-temperature ferromagnetism. The observed saturation magnetic moment and coercivity are better than those of the other TM-doped $\operatorname{In}_{2} \mathrm{O}_{3}$. However, the undoped $\mathrm{In}_{2} \mathrm{O}_{3}$ and $\mathrm{SnO}_{2}$ samples exhibited diamagnetic behaviour as shown, respectively, in figure 7 top and bottom insets, and converted to ferromagnetic behaviour by $\mathrm{Sn}$ doping into the $\mathrm{In}_{2} \mathrm{O}_{3}$ lattice without any magnetic impurities. Hence it is believed that the observed roomtemperature ferromagnetism in ITO was intrinsic rather than any magnetic-impurity-induced one.

Photoluminescence (PL) property of ITO is also very important as it finds applications in the field of lasers, lightemitting diodes, optical sensors, etc. The PL spectra for the ITO nanoparticles were recorded to study the nature of defects present within the ITO lattice. The photoluminescence property of ITO nanostructures was studied by a few research groups, who suggested that the emission mechanism in these nanostructures might be due to bound and free electron exciton emission along with oxygen vacancy/oxygenindium vacancy pairs [30,31]. Recently Kundu and Biswas [32] studied the photoluminescence properties of ITO thin films and reported that the reason for the emissions is bound excitons, which were trapped in the defect centres of ITO. Figure 8 shows the PL spectrum of ITO nanoparticles. These nanoparticles exhibited both UV and blue-green

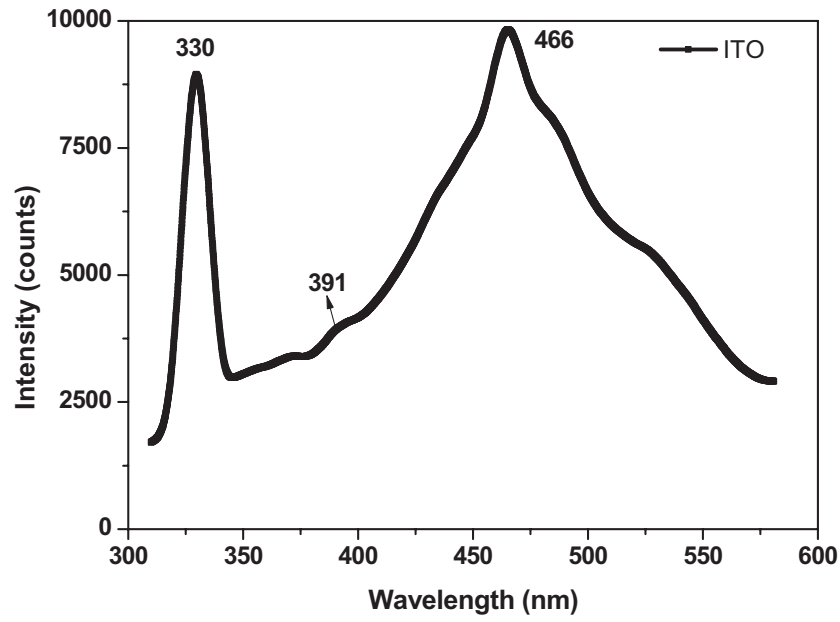

Figure 8. Photoluminescence spectrum of ITO nanoparticles.

emissions. Two broad emission peaks at 330 and $460 \mathrm{~nm}$ were found from ITO nanoparticles. The emission peaks in ultraviolet region around $330 \mathrm{~nm}$, which is very close to that of band gap of $\operatorname{In}_{2} \mathrm{O}_{3}$, is believed to be due to the near-band-edge (NBE) radiative transitions related to the photogenerated electrons [33,34]. From the PL spectrum it is clear that the ITO exhibited much stronger ultraviolet emission peaks at 330 and $466 \mathrm{~nm}$. Here it is expected that the samples prepared in oxygen-deficient environment will have more surface defects and can emit strong UV emission bands in ITO. From figure 3 (FE-SEM image), it can be seen that the particles have large surface area, which contains more defects such as vacancies, or surface defects, which can 
form different defect levels within the ITO, and hence the transition of electrons can give defect emissions at blue and blue-green regimes. Hence here it is believed that the emission bands at blue and blue-green (330-580nm) regions are due to various crystalline defects such as surface defects and oxygen vacancies $[35,36]$.

\section{Conclusions}

The ITO nanoparticles were prepared by solid-state reaction and studied for their structural, chemical, surface, magnetic and PL properties. The tin ( $\mathrm{Sn}$ ) doping into $\mathrm{In}_{2} \mathrm{O}_{3}$ lattice leads to decrease in crystallite size and lattice parameter. These results were supported by the X-ray diffraction pattern along with FE-SEM micrographs. Raman studies indicated the presence of structural disorder and oxygen vacancies present in the system. The ITO nanoparticles exhibited roomtemperature ferromagnetism, and it was found that this was intrinsic in nature rather than due to any external impurities. Blue and blue-green emission was found from the ITO nanoparticles, which could be due to vacancies or surface defects present in the systems.

\section{Acknowledgements}

We are grateful to UGC-DAE-CSR, IGCAR, Kalpakkam 603102, Tamilnadu, India, for providing financial (Grant no. CSR-KN/CRS-72/2015-16/809) support to carry out the present work. We are highly thankful to Dr R K Kotnala, Dr J Shah and Dr G A Bashheed, National Physical Laboratory, India, for providing the vibrating sample magnetometer facilities. We also thank VIT-SIF for providing XRD, Raman and UV-vis-NIR facilities.

\section{References}

[1] Dietl T, Ohno H, Matsukura F, Cibert J and Ferrand D 2000 Science 2871019

[2] Jonker B T, Park Y D, Bennett B R, Cheong H D, Kioseoglou G and Petrou A 2000 Phys. Rev. B 628180

[3] Kaushik A, Dalela B, Kumar S, Alvi P A and Dalela S 2013 J. Alloys Compd. 552274

[4] Saleh R, Djaja N F and Prakoso S P 2013 J. Alloys Compd. 54648

[5] Jlaiel F, Amami M, Boudjada N, Strobel P and Salah A 2011 J. Alloys Compd. $\mathbf{5 0 9} 7784$

[6] Khatoon S, Coolahan K, Lofland S E and Ahmad T 2012 J. Alloys Compd. 545162

[7] Fitzgerald C B, Venkatesan M, Douvalis A P, Huber S, Coey J M D and Bakas T 2004 J. Appl. Phys. 957390

[8] Ahmad T and Khatoon S 2015 J. Mater. Res. 301611
[9] Khatoon S, Coolahan K, Lofland S E and Ahmad T 2013 J. Am. Ceram. Soc. 962544

[10] Gordon R G 2000 MRS Bull. 2552

[11] Ederth J, Hultaker A, Niklasson G A, Heszler P, Doorn A R V, Jongerius M J, Burgard D and Granqvist C G 2005 Appl. Phys. A: Mater. Sci. Process. 811363

[12] Buhler G, Tholmann D and Feldmann C 2007 Adv. Mater. 192224

[13] Prasad K R, Koga K and Miura N 2004 Chem. Mater. 161845

[14] Xia B, Wu Y, Ho H W, Ke C, Song W D, Huan C H A, Kuo J L, Zhu W G and Wang L 2011 Physica B 4063166

[15] Cullity B D 1978 Elements of X-ray diffraction, 2nd edn (Reading, MA: Addison Wesley) p 10

[16] Kachouane A, Addou M, Bougrine A, Elidrissi B, Messoussi R, Regragui M and Bernede J C 2001 Mater. Chem. Phys. 70285

[17] Zhu F, Huan C H A, Zhang K and Wee A T S 2000 Thin Solid Films 359254

[18] Majumdar H S, Majumdar S, Tobjork D and Osterbacka R 2010 Synth. Met. 160303

[19] Ayeshamariam A, Ramalingam S, Bououdina $M$ and Jayachandran M 2014 Spectrochim. Acta Part A 1181135

[20] Shek C H, Lin G M and Lai J K L 1999 Nano Struct. Mater. 11831

[21] Kaur J, Shah J, Kotnala R K and Verma K C 2012 Ceram. Int. 385563

[22] Mcguire K, Pan Z W, Wang Z L, Milkie D, Menendez J and Rao A M 2002 J. Nanosci. Nanotechnol. 21

[23] Zhou W, Liu R, Wan Q, Zhang Q, Pan A L, Guo L and Zou B 2009 J. Phys. Chem. C 1131719

[24] Dussan S, Singh M K, Kumar A and Katiyar S 2011 Integr. Ferroelectr. 125155

[25] Santara B, Pal B and Giri P K 2011 J. Appl. Phys. 110114322

[26] Zener C 1951 Phys. Rev. 81440

[27] Ruderman M A and Kittel C 1954 Phys. Rev. 9699

[28] Coey J M D, Douvalis A P, Fitzgerald C B and Venkatesan M 2004 Appl. Phys. Lett. 841332

[29] Coey J M D, Venkatesan M and Fitzgerald C B 2005 Nat. Mater. 4173

[30] Sun Z, He J, Kumbhar A and Fang J 2010 Langmuir 264246

[31] Choi S I, Nam K M, Park B K, Seo W S and Park J T 2008 Chem. Mater. 202609

[32] Kundu S and Biswas P K 2005 Chem. Phys. Lett. 414107

[33] Khan G G, Ghosh S, Sankar A, Mandal G, Mukherjee G D, Manju U, Banu N and Dev B N 2015 Appl. Phys. Lett. 118074303

[34] Taniguchi T, Yamaguchi K, Shigeta A, Matsuda Y, Hayami S, Shimizu T, Matsui T, Yamazaki T, Funatstu A, Makinose Y, Matsushita N, Koinuma M and Matsumoto Y 2013 Adv. Funct. Mater. 233140

[35] Gao J, Chen R, Li D H, Jiang L, Ye J C, Ma X C, Chen X D, Xiong Q H, Sun H D and Wu T 2011 Nanotechnology 22195706

[36] Hsin C L, He J H and Chen L J 2006 Appl. Phys. Lett. 88063111 